\title{
The Incubation Period of Coronavirus Disease 2019 (COVID-19) From Publicly Reported Confirmed Cases: Estimation and Application
}

\author{
Stephen A. Lauer, MS, PhD*; Kyra H. Grantz, BA*; Qifang Bi, MHS; Forrest K. Jones, MPH; Qulu Zheng, MHS; \\ Hannah R. Meredith, PhD; Andrew S. Azman, PhD; Nicholas G. Reich, PhD; and Justin Lessler, PhD
}

Background: A novel human coronavirus, severe acute respiratory syndrome coronavirus 2 (SARS-CoV-2), was identified in China in December 2019. There is limited support for many of its key epidemiologic features, including the incubation period for clinical disease (coronavirus disease 2019 [COVID-19]), which has important implications for surveillance and control activities.

Objective: To estimate the length of the incubation period of COVID-19 and describe its public health implications.

Design: Pooled analysis of confirmed COVID-19 cases reported between 4 January 2020 and 24 February 2020

Setting: News reports and press releases from 50 provinces, regions, and countries outside Wuhan, Hubei province, China.

Participants: Persons with confirmed SARS-CoV-2 infection outside Hubei province, China.

Measurements: Patient demographic characteristics and dates and times of possible exposure, symptom onset, fever onset, and hospitalization.

Results: There were 181 confirmed cases with identifiable exposure and symptom onset windows to estimate the incubation period of COVID-19. The median incubation period was estimated to be 5.1 days ( $95 \% \mathrm{Cl}, 4.5$ to 5.8 days), and $97.5 \%$ of those who develop symptoms will do so within 11.5 days $(\mathrm{Cl}, 8.2$ to 15.6 days) of infection. These estimates imply that, under conservative assumptions, 101 out of every 10000 cases (99th percentile, 482) will develop symptoms after 14 days of active monitoring or quarantine.

Limitation: Publicly reported cases may overrepresent severe cases, the incubation period for which may differ from that of mild cases.

Conclusion: This work provides additional evidence for a median incubation period for COVID-19 of approximately 5 days, similar to SARS. Our results support current proposals for the length of quarantine or active monitoring of persons potentially exposed to SARS-CoV-2, although longer monitoring periods might be justified in extreme cases.

Primary Funding Source: U.S. Centers for Disease Control and Prevention, National Institute of Allergy and Infectious Diseases, National Institute of General Medical Sciences, and Alexander von Humboldt Foundation.

Ann Intern Med. doi:10.7326/M20-0504

Annals.org

For author affiliations, see end of text.

This article was published at Annals.org on 10 March 2020

* Dr. Lauer and Ms. Grantz share first authorship.
In December 2019, a cluster of severe pneumonia cases of unknown cause was reported in Wuhan, Hubei province, China. The initial cluster was epidemiologically linked to a seafood wholesale market in Wuhan, although many of the initial 41 cases were later reported to have no known exposure to the market (1). A novel strain of coronavirus belonging to the same family of viruses that cause severe acute respiratory syndrome (SARS) and Middle East respiratory syndrome (MERS), as well as the 4 human coronaviruses associated with the common cold, was subsequently isolated from lower respiratory tract samples of 4 cases on 7 January 2020 (2). Infection with the virus, severe acute respiratory syndrome coronavirus 2 (SARS-CoV2), can be asymptomatic or can result in mild to severe symptomatic disease (coronavirus disease 2019 [COVID19]) (3). On 30 January 2020, the World Health Organization declared that the SARS-CoV-2 outbreak constituted a Public Health Emergency of International Concern, and more than 80000 confirmed cases had been reported worldwide as of 28 February 2020 (4, 5). On 31 January 2020, the U.S. Centers for Disease Control and Prevention announced that all citizens returning from Hubei province, China, would be subject to mandatory quarantine for up to 14 days (6).

Our current understanding of the incubation period for COVID-19 is limited. An early analysis based on
88 confirmed cases in Chinese provinces outside Wuhan, using data on known travel to and from Wuhan to estimate the exposure interval, indicated a mean incubation period of 6.4 days ( $95 \% \mathrm{Cl}, 5.6$ to 7.7 days), with a range of 2.1 to 11.1 days (7). Another analysis based on 158 confirmed cases outside Wuhan estimated a median incubation period of 5.0 days $(\mathrm{Cl}, 4.4$ to 5.6 days), with a range of 2 to 14 days (8). These estimates are generally consistent with estimates from 10 confirmed cases in China (mean incubation period, 5.2 days [Cl, 4.1 to 7.0 days] [9]) and from clinical reports of a familial cluster of COVID-19 in which symptom onset occurred 3 to 6 days after assumed exposure in Wuhan (1). These estimates of the incubation period of SARSCoV-2 are also in line with those of other known human coronaviruses, including SARS (mean, 5 days; range, 2 to 14 days [10]), MERS (mean, 5 to 7 days; range, 2 to 14 days [11]), and non-SARS human coronavirus (mean, 3 days; range, 2 to 5 days [12]).

The incubation period can inform several important public health activities for infectious diseases, including active monitoring, surveillance, control, and modeling. Active monitoring requires potentially exposed persons to contact local health authorities to report their health status every day. Understanding the length of active monitoring needed to limit the risk for missing SARS-CoV-2 infections is necessary for health 
departments to effectively use limited resources. In this article, we provide estimates of the incubation period of COVID-19 and the number of symptomatic infections missed under different active monitoring scenarios.

\section{Methods \\ Data Collection}

We searched for news and public health reports of confirmed COVID-19 cases in areas with no known community transmission, including provinces, regions, and countries outside Hubei. We searched for reports in both English and Chinese and abstracted the data necessary to estimate the incubation period of COVID19. Two authors independently reviewed the full text of each case report. Discrepancies were resolved by discussion and consensus.

For each case, we recorded the time of possible exposure to SARS-CoV-2, any symptom onset, fever onset, and case detection. The exact time of events was used when possible; otherwise, we defined conservative upper and lower bounds for the possible interval of each event. For most cases, the interval of possible SARS-CoV-2 exposure was defined as the time between the earliest possible arrival to and latest possible departure from Wuhan. For cases without history of travel to Wuhan but with assumed exposure to an infectious person, the interval of possible SARS-CoV-2 exposure was defined as the maximum possible interval of exposure to the infectious person, including time before the infectious person was symptomatic. We allowed for the possibility of continued exposure within known clusters (for example, families traveling together) when the ordering of transmission was unclear. We assumed that exposure always preceded symptom onset. If we were unable to determine the latest exposure time from the available case report, we defined the upper bound of the exposure interval to be the latest possible time of symptom onset. When the earliest possible time of exposure could not be determined, we defined it as 1 December 2019, the date of symptom onset in the first known case (1); we performed a sensitivity analysis for the selection of this universal lower bound. When the earliest possible time of symptom onset could not be determined, we assumed it to be the earliest time of possible exposure. When the latest time of possible symptom onset could not be determined, we assumed it to be the latest time of possible case detection. Data on age, sex, country of residence, and possible exposure route were also collected.

\section{Statistical Analysis}

Cases were included in the analysis if we had information on the interval of exposure to SARS-CoV-2 and symptom onset. We estimated the incubation time using a previously described parametric accelerated failure time model (13). For our primary analysis, we assumed that the incubation time follows a log-normal distribution, as seen in other acute respiratory viral infections (12). We fit the model to all observations, as well as to only cases where the patient had fever and only those detected inside or outside mainland China in subset analyses. Finally, we also fit 3 other commonly used incubation period distributions (gamma, Weibull, and Erlang). We estimated median incubation time and important quantiles (2.5th, 25th, 75th, and 97.5th percentiles) along with their bootstrapped $\mathrm{Cls}$ for each model.

Using these estimates of the incubation period, we quantified the expected number of undetected symptomatic cases in an active monitoring program, adapting a method detailed by Reich and colleagues (14). We accounted for varying durations of the active monitoring program ( 1 to 28 days) and individual risk for symptomatic infection (low risk: 1-in-10 000 chance of infection; medium risk: 1-in-1000 chance; high risk: 1-in-100 chance; infected: 1-in-1 chance). For each bootstrapped set of parameter estimates from the lognormal model, we calculated the probability of a symptomatic infection developing after an active monitoring program of a given length for a given risk level. This model conservatively assumes that persons are exposed to SARS-CoV-2 immediately before the active monitoring program and assumes perfect ascertainment of symptomatic cases that develop under active monitoring. We report the mean and 99th percentile of the expected number of undetected symptomatic cases for each active monitoring scenario.

All estimates are based on persons who developed symptoms, and this work makes no inferences about asymptomatic infection with SARS-CoV-2. The analyses were conducted using the coarseDataTools and activemonitr packages in the $\mathrm{R}$ statistical programming language, version 3.6.2 (R Foundation for Statistical Computing). All code and data are available at https: //github.com/HopkinsIDD/ncov_incubation (release at time of submission at https://zenodo.org/record/3692048) (15).

\section{Role of the Funding Source}

The findings and conclusions in this manuscript are those of the authors and do not necessarily represent the views of the U.S. Centers for Disease Control and Prevention, the National Institute of Allergy and Infectious Diseases, the National Institute of General Medical Sciences, and the Alexander von Humboldt Foundation. The funders had no role in study design, data collection and analysis, preparation of the manuscript, or the decision to submit the manuscript for publication.

\section{Results}

We collected data from 181 cases with confirmed SARS-CoV-2 infection detected outside Hubei province before 24 February 2020 (Table 1). Of these, 69 (38\%) were female, 108 were male (60\%), and 4 (2\%) were of unknown sex. The median age was 44.5 years (interquartile range, 34.0 to 55.5 years). Cases were collected from 24 countries and regions outside mainland China $(n=108)$ and 25 provinces within mainland China $(n=73)$. Most cases $(n=161)$ had a known re- 


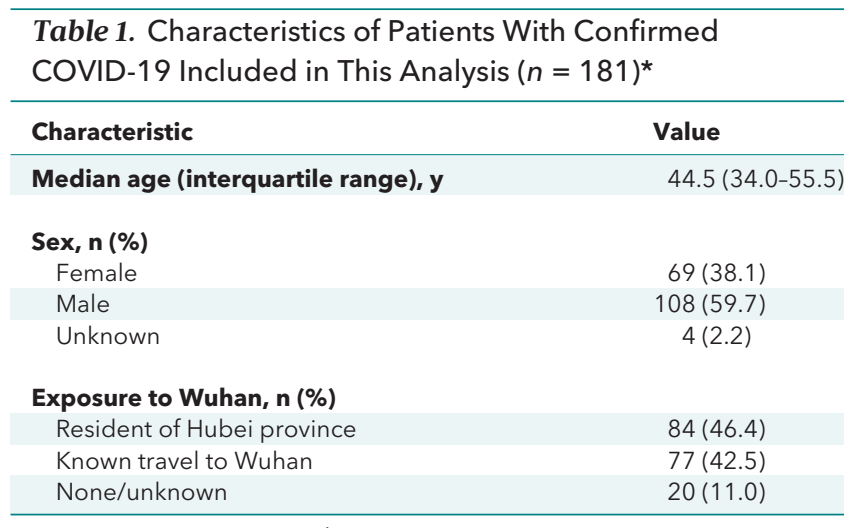

COVID-19 = coronavirus disease 2019.

* Regions of case detection include mainland China $(n=73)$, Singapore $(n=16)$, Japan $(n=13)$, Taiwan $(n=10)$, Hong Kong $(n=8)$, South Korea $(n=8)$, Thailand $(n=8)$, Malaysia $(n=7)$, Australia $(n=6)$, Macau $(n=5)$, the United States $(n=5)$, France $(n=4)$, the Philippines $(n=3)$, Canada $(n=2)$, Italy $(n=2)$, Vietnam $(n=2)$, Brazil $(n=1)$, Cambodia $(n=1)$, Finland $(n=1)$, Germany $(n=1)$, Lebanon $(n=1)$, Nepal $(n=1)$, Sri Lanka $(n=1)$, Sweden $(n=1)$, and the United Arab Emirates $(n=1)$.

cent history of travel to or residence in Wuhan; others had evidence of contact with travelers from Hubei or persons with known infection. Among those who developed symptoms in the community, the median time from symptom onset to hospitalization was 1.2 days (range, 0.2 to 29.9 days) (Figure 1 ).

Fitting the log-normal model to all cases, we estimated the median incubation period of COVID-19 to be 5.1 days $(\mathrm{Cl}, 4.5$ to 5.8 days) (Figure 2). We estimated that fewer than $2.5 \%$ of infected persons will show symptoms within 2.2 days $(\mathrm{Cl}, 1.8$ to 2.9 days) of exposure, and symptom onset will occur within 11.5 days $(\mathrm{Cl}, 8.2$ to 15.6 days) for $97.5 \%$ of infected persons. The estimate of the dispersion parameter was $1.52(\mathrm{Cl}, 1.32$ to 1.72$)$, and the estimated mean incubation period was 5.5 days.

To control for possible bias from symptoms of cough or sore throat, which could have been caused by other more common pathogens, we performed the same analysis on the subset of cases with known time of fever onset $(n=99)$, using the time from exposure to onset of fever as the incubation time. We estimated the median incubation period to fever onset to be 5.7 days $(\mathrm{Cl}, 4.9$ to 6.8 days), with $2.5 \%$ of persons experiencing fever within 2.6 days $(\mathrm{Cl}, 2.1$ to 3.7 days $)$ and $97.5 \%$ having fever within 12.5 days $(\mathrm{Cl}, 8.2$ to 17.7 days $)$ of exposure.

Because assumptions about the occurrence of local transmission and therefore the period of possible exposure may be less firm within mainland China, we also analyzed only cases detected outside mainland China $(n=108)$. The median incubation period for these cases was 5.5 days $(\mathrm{Cl}, 4.4$ to 7.0 days), with the $95 \%$ range spanning from $2.1(\mathrm{Cl}, 1.5$ to 3.2$)$ to $14.7(\mathrm{Cl}, 7.4$ to 22.6) days. Alternatively, persons who left mainland China may represent a subset of persons with longer incubation periods, persons who were able to travel internationally before symptom onset within China, or persons who may have chosen to delay reporting

\section{Figure 1. SARS-CoV-2 exposure (blue), symptom onset (red), and case detection (green) times for 181 confirmed cases.}

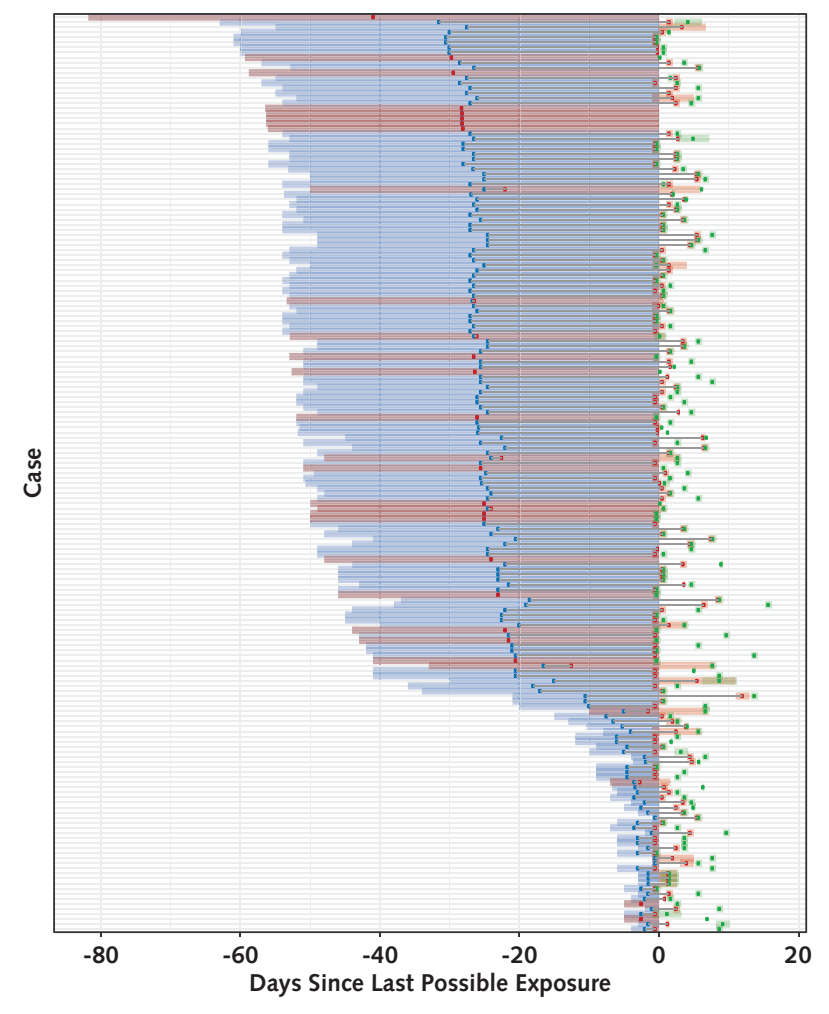

Shaded regions represent the full possible time intervals for exposure, symptom onset, and case detection; points represent the midpoints of these intervals. SARS-CoV- $2=$ severe acute respiratory syndrome coronavirus 2.

Figure 2. Cumulative distribution function of the COVID-19 incubation period estimate from the log-normal model.

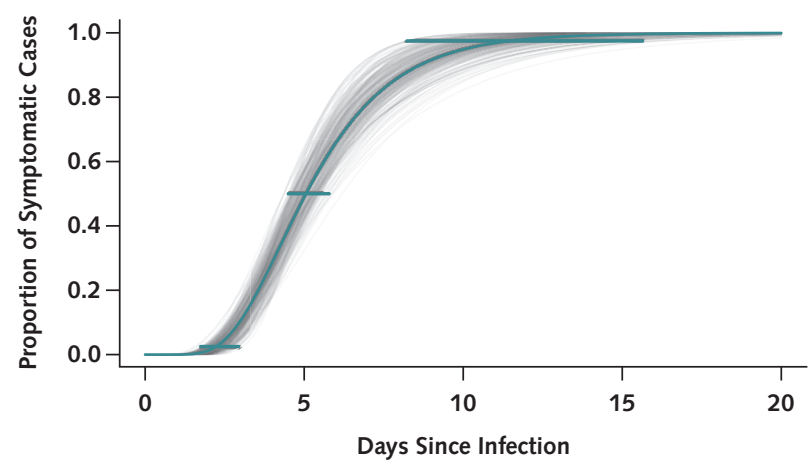

The estimated median incubation period of COVID-19 was 5.1 days ( $\mathrm{Cl}, 4.5$ to 5.8 days). We estimated that fewer than $2.5 \%$ of infected persons will display symptoms within 2.2 days $(\mathrm{Cl}, 1.8$ to 2.9 days) of exposure, whereas symptom onset will occur within 11.5 days $(\mathrm{Cl}, 8.2$ to 15.6 days) for $97.5 \%$ of infected persons. Horizontal bars represent the $95 \% \mathrm{Cls}$ of the 2.5 th, 50 th, and 97.5 th percentiles of the incubation period distribution. The estimate of the dispersion parameter is 1.52 (Cl, 1.32 to 1.72). COVID-19 = coronavirus disease 2019 . 
Table 2. Expected Number of Symptomatic SARS-CoV-2 Infections That Would Be Undetected During Active Monitoring, Given Varying Monitoring Durations and Risks for Symptomatic Infection After Exposure*

\begin{tabular}{|c|c|c|c|c|}
\hline \multirow[t]{2}{*}{$\begin{array}{l}\text { Monitoring } \\
\text { Duration }\end{array}$} & \multicolumn{4}{|c|}{$\begin{array}{l}\text { Mean Estimated Number of Undetected Symptomatic } \\
\text { Infections per } 10000 \text { Monitored Persons (99th Percentile) }\end{array}$} \\
\hline & $\begin{array}{l}\text { Low Risk } \\
(1 \text { in } 10000)\end{array}$ & $\begin{array}{l}\text { Medium Risk } \\
(1 \text { in 1000) }\end{array}$ & $\begin{array}{l}\text { High Risk } \\
(1 \text { in 100) }\end{array}$ & $\begin{array}{l}\text { Infected } \\
(1 \text { in } 1)\end{array}$ \\
\hline $7 d$ & $0.2(0.4)$ & $2.1(3.6)$ & $21.2(36.5)$ & $2120.6(3648.5)$ \\
\hline $14 d$ & $0.0(0.0)$ & $0.1(0.5)$ & $1.0(4.8)$ & $100.9(481.7)$ \\
\hline $21 \mathrm{~d}$ & $0.0(0.0)$ & $0.0(0.1)$ & $0.1(0.8)$ & $9.5(82.5)$ \\
\hline $28 d$ & $0.0(0.0)$ & $0.0(0.0)$ & $0.0(0.2)$ & $1.4(17.8)$ \\
\hline
\end{tabular}

SARS-CoV-2 = severe acute respiratory syndrome coronavirus 2.

* Estimates were generated from a probabilistic model using the incubation period estimates from the log-normal model.

symptoms until they left China. Based on cases detected inside mainland China $(n=73)$, the median incubation period is 4.8 days ( $\mathrm{Cl}, 4.2$ to 5.6 days), with a $95 \%$ range of $2.5(\mathrm{Cl}, 1.9$ to 3.5$)$ to $9.2(\mathrm{Cl}, 6.4$ to 12.5$)$ days. Full results of these sensitivity analyses are presented in Appendix Table 1 (available at Annals.org).

We fit other commonly used parameterizations of the incubation period (gamma, Weibull, and Erlang distributions). The incubation period estimates for these alternate parameterizations were similar to those from the log-normal model (Appendix Table 2, available at Annals.org).

Given these estimates of the incubation period, we predicted the number of symptomatic infections we would expect to miss over the course of an active monitoring program. We classified persons as being at high risk if they have a 1 -in-100 chance of developing a symptomatic infection after exposure. For an active monitoring program lasting 7 days, the expected number of symptomatic infections missed for every 10000 high-risk persons monitored is 21.2 (99th percentile, 36.5) (Table 2 and Figure 3). After 14 days, it is highly unlikely that further symptomatic infections would be undetected among high-risk persons (mean, 1.0 undetected infections per 10000 persons [99th percentile, 4.8]). However, substantial uncertainty remains in the classification of persons as being at "high," "medium," or "low" risk for being symptomatic, and this method does not consider the role of asymptomatic infection. We have created an application to estimate the proportion of missed COVID-19 cases across any active monitoring duration up to 100 days and various population risk levels (16).

\section{Discussion}

We present estimates of the incubation period for the novel coronavirus disease (COVID-19) that emerged in Wuhan, Hubei province, China, in 2019. We estimated the median incubation period of COVID-19 to be 5.1 days and expect that nearly all infected persons who have symptoms will do so within 12 days of infection. We found that the current period of active monitoring recommended by the U.S. Centers for Disease Control and Prevention (14 days) is well supported by the evidence (6). Symptomatic disease is frequently associated with transmissibility of a patho- gen. However, given recent evidence of SARS-CoV-2 transmission by mildly symptomatic and asymptomatic persons $(17,18)$, we note that time from exposure to onset of infectiousness (latent period) may be shorter than the incubation period estimated here, with important implications for transmission dynamics.

Our results are broadly consistent with other estimates of the incubation period (1, 7-9). Our analysis, which was based on 181 confirmed COVID-19 cases, made more conservative assumptions about the possible window of symptom onset and the potential for continued exposure through transmission clusters outside Wuhan. Of note, the use of fixed times of symptom onset, as used in 3 of the 4 prior analyses, will truncate the incubation period distribution by either decreasing the maximum possible incubation period (if the earliest possible time of symptom onset is used) or increasing the minimum possible incubation period (if the midpoint or latest possible time of symptom onset is used). Therefore, using a symptom onset window more accu-

Figure 3. Proportion of known symptomatic SARS-CoV-2 infections that have yet to develop symptoms, by number of days since infection, using bootstrapped estimates from a log-normal accelerated failure time model.

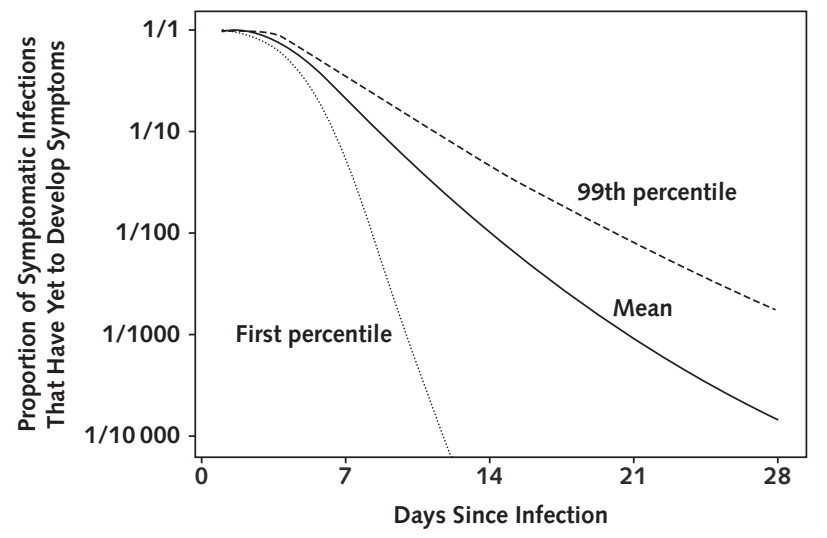

The solid line represents the mean estimate, the dashed line represents the 99th percentile estimate, and the dotted line represents the first percentile estimate. See Table 2 for exact estimates at various time points and at different levels of population risk for symptomatic infection. SARS-CoV-2 = severe acute respiratory syndrome coronavirus 2 
rately accounts for the full distribution of possible incubation periods.

Although our results support current proposals for the length of quarantine or active monitoring of persons potentially exposed to SARS-CoV-2, longer monitoring periods might be justified in extreme cases. Among those who are infected and will develop symptoms, we expect 101 in 10000 (99th percentile, 482) will do so after the end of a 14-day monitoring period (Table 2 and Figure 3), and our analyses do not preclude this estimate from being higher. Although it is essential to weigh the costs of extending active monitoring or quarantine against the potential or perceived costs of failing to identify a symptomatic case, there may be high-risk scenarios (for example, a health care worker who cared for a COVID-19 patient while not wearing personal protective equipment) where it could be prudent to extend the period of active monitoring.

This analysis has several important limitations. Our data include early case reports, with associated uncertainty in the intervals of exposure and symptom onset. We have used conservative bounds of possible exposure and symptom onset where exact times were not known, but there may be further inaccuracy in these data that we have not considered. We have exclusively considered reported, confirmed cases of COVID-19, which may overrepresent hospitalized persons and others with severe symptoms, although we note that the proportion of mild cases detected has increased as surveillance and monitoring systems have been strengthened. The incubation period for these severe cases may differ from that of less severe or subclinical infections and is not typically an applicable measure for those with asymptomatic infections.

Our model assumes a constant risk for SARS-CoV-2 infection in Wuhan from 1 December 2019 to 30 January 2020, based on the date of symptom onset of the first known case and the last known possible exposure within Wuhan in our data set. This is a simplification of infection risk, given that the outbreak has shifted from a likely common-source outbreak associated with a seafood market to human-to-human transmission. Moreover, phylogenetic analysis of 38 SARS-CoV-2 genomes suggests that the virus may have been circulating before December 2019 (19). To test the sensitivity of our estimates to that assumption, we performed an analysis where cases with unknown lower bounds on exposure were set to 1 December 2018, a full year earlier than in our primary analysis. Changing this assumption had little effect on the estimates of the median ( 0.2 day longer than for the overall estimate) and the 97.5th quantile ( 0.1 day longer) of the incubation period. In data sets such as ours, where we have adequate observations with well-defined minimum and maximum possible incubation periods for many cases, extending the universal lower bound has little bearing on the overall estimates.

This work provides additional evidence for a median incubation period for COVID-19 of approximately 5 days, similar to SARS. Assuming infection occurs at the initiation of monitoring, our estimates suggest that
101 out of every 10000 cases will develop symptoms after 14 days of active monitoring or quarantine. Whether this rate is acceptable depends on the expected risk for infection in the population being monitored and considered judgment about the cost of missing cases (14). Combining these judgments with the estimates presented here can help public health officials to set rational and evidence-based COVID-19 control policies.

From Johns Hopkins Bloomberg School of Public Health, Baltimore, Maryland (S.A.L., K.H.G., Q.B., F.K.J., Q.Z., H.R.M., A.S.A., J.L.); and School of Public Health and Health Sciences, University of Massachusetts, Amherst, Massachusetts, and Ludwig-Maximilians-Universität, Munich, Germany (N.G.R.).

Acknowledgment: The authors thank all who have collected, prepared, and shared data throughout this outbreak. They are particularly grateful to Dr. Kaiyuan Sun, Ms. Jenny Chen, and Dr. Cecile Viboud from the Division of International Epidemiology and Population Studies, Fogarty International Center, National Institutes of Health; Dr. Moritz Kraemer and the open COVID-19 data working group; and the Johns Hopkins Center for Systems Science and Engineering.

Grant Support: By the U.S. Centers for Disease Control and Prevention (NU2GGH002000), the National Institute of Allergy and Infectious Diseases (R01 Al135115), the National Institute of General Medical Sciences (R35 GM119582), and the Alexander von Humboldt Foundation.

Disclosures: Dr. Lauer reports grants from the National Institute of Allergy and Infectious Diseases and the U.S. Centers for Disease Control and Prevention during the conduct of the study. Ms. Grantz reports a grant from the U.S. Centers for Disease Control and Prevention during the conduct of the study. Dr. Reich reports grants from the National Institute of General Medical Sciences and the Alexander von Humboldt Foundation during the conduct of the study. Dr. Lessler reports a grant from the U.S. Centers for Disease Control and Prevention during the conduct of the study. Authors not named here have disclosed no conflicts of interest. Disclosures can also be viewed at www.acponline.org/authors/icmje /ConflictOfInterestForms.do?msNum=M20-0504.

Reproducible Research Statement: Study protocol: Not applicable. Statistical code and data set: Available at https://github .com/HopkinsIDD/ncov_incubation.

Corresponding Author: Justin Lessler, PhD, Department of Epidemiology, Bloomberg School of Public Health, Johns Hopkins University, 615 North Wolfe Street, Baltimore, MD 21205; e-mail, justin@jhu.edu.

Previous Posting: This manuscript was posted as a preprint on medRxiv on 4 February 2020. doi:10.1101/2020.02.02 .20020016

Current author addresses and author contributions are available at Annals.org. 


\section{References}

1. Huang $C$, Wang $Y$, Li $X$, et al. Clinical features of patients infected with 2019 novel coronavirus in Wuhan, China. Lancet. 2020;395:497506. [PMID: 31986264] doi:10.1016/S0140-6736(20)30183-5

2. Zhu N, Zhang D, Wang W, et al; China Novel Coronavirus Investigating and Research Team. A novel coronavirus from patients with pneumonia in China, 2019. N Engl J Med. 2020;382:727-733. [PMID: 31978945] doi:10.1056/NEJMoa2001017

3. The Novel Coronavirus Pneumonia Emergency Response Epidemiology Team. The Epidemiological Characteristics of an Outbreak of 2019 Novel Coronavirus Diseases (COVID-19)-China, 2020. China CDC Weekly. 2020;2:113-22.

4. World Health Organization. Coronavirus disease 2019 (COVID19): Situation Report - 38. 27 February 2020. Accessed at www.who int/docs/default-source/coronaviruse/situation-reports/20200227 -sitrep-38-covid-19.pdf?sfvrsn=9f98940c_2 on 28 February 2020.

5 . World Health Organization. Statement on the second meeting of the International Health Regulations (2005) Emergency Committee regarding the outbreak of novel coronavirus (2019-nCoV). 30 January 2020. Accessed at www.who.int/news-room/detail/30-01-2020 -statement-on-the-second-meeting-of-the-international-health -regulations-(2005)-emergency-committee-regarding-the-outbreak -of-novel-coronavirus-(2019-ncov) on 31 January 2020.

6. The White House. Press Briefing by Members of the President's Coronavirus Task Force. 31 January 2020. Accessed at www.whitehouse.gov /briefings-statements/press-briefing-members-presidents-coronavirus -task-force on 1 February 2020.

7. Backer JA, Klinkenberg D, Wallinga J. Incubation period of 2019 novel coronavirus (2019-nCoV) infections among travellers from Wuhan, China, 20-28 January 2020. Euro Surveill. 2020;25. [PMID: 32046819] doi:10.2807/1560-7917.ES.2020.25.5.2000062

8. Linton NM, Kobayashi T, Yang Y, et al. Incubation period and other epidemiological characteristics of 2019 novel coronavirus infections with right truncation: a statistical analysis of publicly available case data. J Clin Med. 2020;9. [PMID: 32079150] doi:10.3390 /jcm9020538
9. Li Q, Guan X, Wu P, et al. Early transmission dynamics in Wuhan, China, of novel coronavirus-infected pneumonia. N Engl J Med. 2020. [PMID: 31995857] doi:10.1056/NEJMoa2001316

10. Varia M, Wilson S, Sarwal S, et al; Hospital Outbreak Investigation Team. Investigation of a nosocomial outbreak of severe acute respiratory syndrome (SARS) in Toronto, Canada. CMAJ. 2003;169: 285-92. [PMID: 12925421]

11. Virlogeux V, Fang VJ, Park $M$, et al. Comparison of incubation period distribution of human infections with MERS-CoV in South Korea and Saudi Arabia. Sci Rep. 2016;6:35839. [PMID: 27775012] doi: 10.1038/srep35839

12. Lessler J, Reich NG, Brookmeyer R, et al. Incubation periods of acute respiratory viral infections: a systematic review. Lancet Infect Dis. 2009;9:291-300. [PMID: 19393959] doi:10.1016/S1473-3099 (09)70069-6

13. Reich NG, Lessler J, Cummings DA, et al. Estimating incubation period distributions with coarse data. Stat Med. 2009;28:2769-84. [PMID: 19598148] doi:10.1002/sim.3659

14. Reich NG, Lessler J, Varma JK, et al. Quantifying the risk and cost of active monitoring for infectious diseases. Sci Rep. 2018;8:1093. [PMID: 29348656] doi:10.1038/s41598-018-19406-x

15. Lauer SA, Grantz $\mathrm{KH}, \mathrm{Bi}$ Q, et al. Estimating the incubation time of the novel coronavirus (COVID-19) based on publicly reported cases using coarse data tools. 2020. Accessed at https://github.com /HopkinsIDD/ncov_incubation on 3 March 2020.

16. Determining Durations for Active Monitoring. Accessed at https: //iddynamics.jhsph.edu/apps/shiny/activemonitr on 28 February 2020.

17. Chan JF, Yuan S, Kok KH, et al. A familial cluster of pneumonia associated with the 2019 novel coronavirus indicating person-toperson transmission: a study of a family cluster. Lancet. 2020;395: 514-523. [PMID: 31986261] doi:10.1016/S0140-6736(20)30154-9 18. Rothe $C$, Schunk $M$, Sothmann $P$, et al. Transmission of 2019$\mathrm{nCoV}$ infection from an asymptomatic contact in Germany [Letter]. N Engl J Med. 2020. [PMID: 32003551] doi:10.1056/NEJMc2001468 19. Genomic epidemiology of novel coronavirus (HCoV-19). 2020. Accessed at https://nextstrain.org/ncov on 29 January 2020. 
Current Author Addresses: Drs. Lauer, Meredith, and Lessler; Ms. Grantz; Ms. Bi; Mr. Jones; and Ms. Zheng: Department of Epidemiology, Bloomberg School of Public Health, Johns Hopkins University, 615 North Wolfe Street, Baltimore, MD 21205.

Dr. Azman: Médecins Sans Frontières, Rue de Lausanne 72, 1202 Genève, Switzerland.

Dr. Reich: Department of Biostatistics and Epidemiology, Amherst School of Public Health and Health Sciences, University of Massachusetts, 715 North Pleasant Street, Amherst, MA 01003-9304.
Author Contributions: Conception and design: S.A. Lauer K.H. Grantz, F.K. Jones, N.G. Reich, J. Lessler.

Analysis and interpretation of the data: S.A. Lauer, K.H. Grantz, Q. Bi, F.K. Jones, N.G. Reich, J. Lessler.

Drafting of the article: S.A. Lauer, K.H. Grantz, Q. Bi, F.K. Jones, A.S. Azman, N.G. Reich.

Critical revision of the article for important intellectual content: Q. Bi, F.K. Jones, A.S. Azman, N.G. Reich, J. Lessler.

Final approval of the article: S.A. Lauer, K.H. Grantz, Q. Bi, F.K. Jones, Q. Zheng, H.R. Meredith, A.S. Azman, N.G. Reich, J. Lessler.

Statistical expertise: Q. Bi, N.G. Reich, J. Lessler. Collection and assembly of data: S.A. Lauer, K.H. Grantz, Q. Bi, F.K. Jones, Q. Zheng, H.R. Meredith.

\begin{tabular}{|c|c|c|c|c|c|}
\hline \multicolumn{6}{|c|}{ Appendix Table 1. Percentiles of SARS-CoV-2 Incubation Period From Selected Sensitivity Analyses* } \\
\hline \multirow[t]{2}{*}{ Analysis } & \multicolumn{5}{|c|}{ Incubation Period $(95 \% \mathrm{Cl}), d$} \\
\hline & 2.5th Percentile & 25th Percentile & 50th Percentile & 75th Percentile & 97.5th Percentile \\
\hline Main $(n=181)$ & $2.2(1.8-2.9)$ & $3.8(3.3-4.4)$ & $5.1(4.5-5.8)$ & $6.7(5.7-7.9)$ & $11.5(8.2-15.6)$ \\
\hline Fever $(n=99)$ & $2.6(2.1-3.7)$ & $4.4(3.8-5.2)$ & $5.7(4.9-6.8)$ & $7.5(6.0-9.2)$ & $12.5(8.2-17.7)$ \\
\hline Non-mainland $(n=108)$ & $2.1(1.5-3.2)$ & $3.9(3.2-5.1)$ & $5.5(4.4-7.0)$ & $7.7(5.4-10.3)$ & $14.7(7.4-22.6)$ \\
\hline Mainland $(n=73)$ & $2.5(1.9-3.5)$ & $3.8(3.3-4.6)$ & $4.8(4.2-5.6)$ & $6.0(4.9-7.1)$ & $9.2(6.4-12.5)$ \\
\hline
\end{tabular}

SARS-CoV-2 $=$ severe acute respiratory syndrome coronavirus 2 .

* Using the log-normal model, we estimated the incubation period for several subsets. The first analysis looked at time to fever onset, as opposed to time to first symptom onset as used in the main analysis. The second and third analyses estimated the incubation period for cases outside and within mainland China, respectively. To test the effect of our assumption that the exposure window for Wuhan residents started on 1 December 2019, we used an extreme assumption that the exposure window instead started on 1 December 2018 (EL-2018) and found little change in our estimates.

\begin{tabular}{|c|c|c|}
\hline \multicolumn{3}{|c|}{$\begin{array}{l}\text { Appendix Table 2. Parameter Estimates for Various } \\
\text { Parametric Distributions of the Incubation Period of } \\
\text { SARS-CoV-2 Using } 181 \text { Confirmed Cases* }\end{array}$} \\
\hline \multirow[t]{2}{*}{ Distribution } & \multicolumn{2}{|c|}{ Estimate $(95 \% \mathrm{Cl})$} \\
\hline & Parameter 1 & Parameter 2 \\
\hline Log-normal & $1.621(1.504-1.755)$ & $0.418(0.271-0.542)$ \\
\hline Gamma & $5.807(3.585-13.865)$ & $0.948(0.368-1.696)$ \\
\hline Weibull & $2.453(1.917-4.171)$ & $6.258(5.355-7.260)$ \\
\hline Erlang & $6(3-11)$ & $0.880(0.484-1.895)$ \\
\hline
\end{tabular}

